\title{
Archivos de Cardiología de México

\section{En torno a los orígenes, la evolución y las irradiaciones de la cardiología mexicana}

\section{Alfredo de Micheli*}

Instituto Nacional de Cardiología Ignacio Chávez, México DF, México

Recibido el 13 de agosto de 2013; aceptado el 24 de octubre de 2013

\author{
PALABRAS CLAVE \\ Cardiología \\ mexicana; \\ Hipertensión arterial; \\ Esfigmomanómetros; \\ Terapia metabólica; \\ México
}

\begin{abstract}
Resumen La cardiología mexicana se inició en el siglo XIX con las primeras hazañas de cirujanos y médicos relacionados con las academias locales y con la Escuela de Medicina, creada en 1833 por iniciativa del Dr. Valentín Gómez Farías. Así, el Dr. Manuel Carpio, primer catedrático de Fisiología en dicha Escuela, tradujo y publicó en 1823 el artículo Sobre el uso del pectoriloquo del médico francés Marat, y realizó algunos experimentos sobre la actividad motora del corazón. En la época del segundo imperio, el médico austriaco Samuel von Basch, miembro correspondiente de nuestra Academia de Medicina, realizó estudios que le llevaron a definir la existencia de la hipertensión arterial esencial, por él denominada «aterosclerosis latente». Más aún, regresado a su patria, ideó en 1880 un esfigmomanómetro de columna de mercurio que sirvió de modelo al instrumento construido por Scipione Riva-Rocci y presentado en 1896. Y, en nuestra época, Demetrio Sodi Pallares sistematizó una modalidad de terapia metabólica, que anunció en 1961 bajo la denominación de terapéutica polarizante, i.e. capaz de repolarizar las células cardiacas parcialmente despolarizadas por efecto de la hipoxia o por agresiones directas. De este modo se dieron los primeros pasos en México por derroteros prometedores de muchos éxitos futuros y se inició la gran aventura de la cardioangiología mexicana.

(C) 2013 Instituto Nacional de Cardiología Ignacio Chávez. Publicado por Masson Doyma México S.A. Todos los derechos reservados.
\end{abstract}

\section{About the origin, evolution and irradiation of the Mexican cardiology}

\begin{abstract}
The Mexican cardioangiology started in the nineteen century thanks to first endeavors of surgeons and physicians related to local academies and to School of Medicine, established in 1833 by Dr. Valentin Gómez Farías. Dr. Manuel Carpio, the future first head of department of physiology in this school, translated to Spanish language and published, in 1823, the article On pectoriloquo of the French physician Marat and later performed some experiments on the heart' motion. During the Secont Empire (1864-1867), the physician Samuel von Basch performed studies to define the arterial hypertension, called by him "latent atherosclerosis", i.e.
\end{abstract}

\footnotetext{
* Instituto Nacional de Cardiología Ignacio Chávez México, Juan Badiano N. ${ }^{\circ} .1$ Col. Sección XVI Tlalpan 14080 México, DF. Teléfono: +5255557329 11x1310.

Correo electrónico: alessandro.micheli@cardiologia.org.mx
} 
the "essential hypertension"'. Once he had returned to his country, he invented in 1880, a sphygmomanometer of mercury column, that was the model for the instrument constructed by the Italian physician Scipione Riva-Rocci and presented in 1896. In our time, Dr. Demetrio Sodi Pallares systematized a metabolic therapy called "polarizing therapy", i.e. capable of repolarizing the heart's cells partly depolarized due to hypoxia or direct aggressions. These were the first steps in Mexico on the way to a promising medicine starting and the great adventure of Mexican cardiology.

(c) 2013 Instituto Nacional de Cardiología Ignacio Chávez. Published by Masson Doyma México S.A. All rights reserved.

\section{Antecedentes}

Debe tenerse presente que, en la primera mitad del siglo llamado de las luces, la doctrina circulatoria era conocida en México (entonces Nueva España), en donde había sido descrita claramente por el Dr. Marcos Joseph Salgado, seguidor de Harvey y titular de la cátedra de «Prima de Medicina» en la Real y Pontificia Universidad mexicana ${ }^{1}$. Dicha doctrina fue adoptada favorablemente por esta Universidad que, en el siglo anterior, había tenido una orientación más bien galénica ${ }^{2}$. Y, en la segunda mitad del siglo mencionado, se había introducido aquí la electrología por el padre oratoriano Juan Benito Díaz de Gamarra y Dávalos gracias a la publicación de su libro Elementa recentioris philosophiae $(1774)^{3}$ y de las tesis sustentadas por sus discípulos.

Los primeros pasos en los dominios de la cardioangiología mexicana se dieron principalmente por cirujanos en la primera mitad del siglo XIX. Pero cabe tener presente que el médico veracruzano Manuel Carpio, catedrático de fisiología de la Escuela de Medicina creada en 1833, ya había traducido del francés, y publicado en 1823, el artículo Sobre el uso del pectoriloquo, i.e. del estetoscopio, debido al Dr. Marat ${ }^{4}$. Dicho escrito había sido publicado originalmente en el tomo 40 del Dictionnaire des Sciences Médicales (París, 1819). Y, en los últimos años de su profesorado, Carpio efectuó también algunos experimentos, con la ayuda de Manuel Soriano, para tratar de esclarecer si, de acuerdo a las ideas de Magendie, el primer ruido cardiaco se debía realmente al «choque de catapulta» de los ventrículos contra la pared torácica. En realidad, estas eran simples demostraciones para los estudiantes de medicina. De todos modos, el verdadero difusor del uso del estetoscopio en la República Mexicana fue el Dr. Miguel F. Jiménez. Como relata en sus lecciones ${ }^{5}$, gracias a la auscultación mediata él pudo diagnosticar la insuficiencia aórtica en un hombre de 35 años, quien se quejaba de tos seca persistente y presentaba edema en los miembros inferiores. Jiménez oía «un soplo áspero - ruido de carda - con su máxima intensidad arriba y afuera de la tetilla izquierda. Dicho soplo coincidía con el segundo movimiento, i.e., con la diástole ventricular». Podía inferir, por tanto, que la lesión se localizaba en el orificio aórtico, correspondiente al punto de auscultación, y que se debía a insuficiencia de las sigmoideas aórticas. El examen anatómico de este paciente, efectuado por el propio Dr. Jiménez, reveló una destrucción de las válvulas aórticas y la presencia de concreciones fibrosas adheridas al endocardio del ventrículo izquierdo. Él opinaba que tales alteraciones eran responsables del soplo y de sus características.

Por otra parte, el cirujano alemán Ernesto Hegewish, en noviembre de 1829 , había realizado en la ciudad de Oaxaca la primera ligadura de la carótida común por un aneurisma aparentemente de origen luético ${ }^{6}$. El 13 de noviembre de 1843 el Dr. Rafael Lucio realizó en la capital de la República -al parecer por primera vez en el mundo- la ligadura de la arteria femoral en el vértice inferior del triángulo de Scarpa, por un aneurisma espontáneo de la arteria poplítea derecha. Los doctores Matías Beistegui y Francisco Vertiz efectuaron en 1845 la primera transfusión sanguínea mediante el procedimiento de James Blundell en una paciente con hemorragia puerperal. Y, en 1862, el Dr. Joaquín Blengio Molina aplicó en Campeche la primera inyección hipodérmica (de morfina). En el periodo de la intervención francesa, el 12 de enero de 1864 el cirujano galo Aronsohn hizo en Aguascalientes la primera ligadura, efectuada en América, de la arteria axilar de un torero, desgarrada por una cornada. Por su lado, el Dr. Francisco de Paula Marín introdujo en México la jeringa de Pravaz y el cirujano Luis G. Muñoz, autor de la primera anestesia clorofórmica en la República Mexicana, el 26 de septiembre de 1865 ligó la arteria ilíaca externa de un hombre por un aneurisma inguinal izquierdo. Parece justo mencionar también algunas obras de carácter cardiológico existentes en la biblioteca de la Real y Pontificia Universidad de México y en otras. Entre los autores del siglo xv representados en la biblioteca universitaria, destaca el humanista italiano Nicolás de Lonigo (Leoniceno) con su ensayo critico Plinii et aliorum auctorum qui simplicibus medicaminibus sumpserunt errores notati (incunable procedente de Ferrara, 1492). Tal escrito comprueba que la cultura humanística no era tan autoritaria como se cree. Había publicaciones de autores renacentistas como Pedro de Arévalo con: De cordis structura. Motu... una edición italiana del célebre tratado anatómico del flamenco Andreas Vesalio (Venecia, 1568); otra de Charles de l'Écluse (Carolus Clusius): Exoticorum libri decem, Leiden, 1605; otra del italiano Gerolamo Mercuriali: De arte gymnastica, Venecia, 1601, etc. Se hallaba igualmente el compendio de los apuntes del protomédico Francisco Hernández, elaborado por el Dr. Antonio Recchi (Roma, 1651), así como el tratado de Albrecht von Haller: Elementa physiologiae corporis humani, 8 Vols. 1757-1776. En este se afirma, con base 
en observaciones experimentales, que «el corazón late de manera espontánea e independiente de conexiones nerviosas u otras, de acuerdo con la teoría de la irritabilidad intrínseca». A estos se les agregaría el texto fundamental de J.B. Sénac Traité de la structure du coeur ${ }^{7}$.

Durante el siglo XIX se efectuaron ulteriores adquisiciones de libros. En una, efectuada en 1857, estaba el Traité clinique des maladies du coeur del Dr. Jean Bouillaud ${ }^{8}$, pionero de los estudios sobre la enfermedad reumática, que los franceses denominan aún «Maladie de Bouillaud». Tras la desintegración del efímero segundo imperio, prosiguieron los adelantos médicos y quirúrgicos en el campo de la cardiología bajo los auspicios de la flamante Academia de Medicina. El 19 de diciembre de 1868, el cirujano yucateco Crescencio Boves, en Tacubaya, ligó con éxito la arteria cubital, en su tercio medio superior, por un aneurisma difuso debido a una herida provocada por un instrumento punzante. Por su parte, el Dr. López Muñoz, quien fuera discípulo del Dr. Ignacio Alvarado sucesor de Manuel Carpio en la cátedra de Fisiología de la Escuela de Medicina, en el periodo 1860$1877^{9}$, dejó un relato de los resultados de las «disecciones y experimentaciones que su maestro había emprendido acerca de la circulación sanguínea en las paredes miocárdicas en 1875». En realidad Alvarado estaba repitiendo, sin saberlo, argumentos y observaciones que se debatían desde 1689 , cuando Giovan Battista Scaramucci planteó su hipótesis. Según él los vasos coronarios profundos resultan exprimidos durante la contracción de las fibras miocárdicas que los rodean y, en la diástole, reciben sangre de la aorta. Tras dejar su cátedra, Alvarado redactó asimismo un ensayo acerca de las relaciones entre la circulación y la producción del calor orgánico. Él circunscribía su investigación a «la relación entre el número de pulsaciones y los grados de calor». Infortunadamente no utilizó datos obtenidos de personas normales, sino que se sirvió de los publicados por Liebermeister y por Lorain. Pero amerita recordarse que el fisiólogo Ignacio Alvarado en 1872 había tratado de aliviar los dolores anginosos, debidos a una cardiopatía isquémica aguda, del entonces presidente Benito Juárez mediante aplicaciones de calor sobre su pecho.

\section{Publicaciones nacionales}

En 1870 salió a la luz una tesis recepcional sobre un tema cardiológico, que había sido elaborada en la Escuela de Medicina por el Dr. Mariano Carrillo ${ }^{10}$. Siguieron pronto las primeras tesis concernientes a la digital $(1872)^{11}$, en las que se daba a conocer la acción terapéutica de la digitalina Nativelle. Años más tarde (1878) apareció una tesis enfocada sobre la semiología de las valvulopatías cardiacas y aórticas $^{12}$. Respecto a la publicación de artículos cardiológicos en revistas y libros nacionales, debe mencionarse que las revistas de las antiguas academias locales, precursoras de la Academia Nacional de Medicina, publicaron artículos sobre el tema que estamos considerando. Así, en el tomo Iv (1839) del «Periódico de la Academia de Medicina de México»-i.e la primera academia médica establecida en 1836 en el Hospital de Betlemitas - se hallaba el escrito Angina de pecho, de L. Blanquière. El tomo I de la segunda época de dicha revista (1843) contenía el artículo Enfermedades del corazón, de Gendrín, y el tomo correspondiente al año 1851 acogía el ensayo Hipertrofia del corazón debido al Dr. Juan Nepomuceno Navarro. El «Periódico de la Sociedad Filoiátrica» (1844-1845) proporciona un informe del Dr. Rafael Lucio, «Aneurisma de la arteria poplítea curado por ligadura de la femoral en su parte media», así como una nota del Dr. M. F. Jiménez, «Apuntes sobre la arteritis». Y el primer tomo de «La Unión Médica de México» (1856-1858) exhibía el ensayo Del círculo senil y su relación con la formación grasosa del corazón, del Dr. M. Alfaro. Asimismo en los primeros volúmenes de la «Gaceta Médica de México», que es todavía el órgano de la Academia Nacional de Medicina, y en el periodo 1864-1870 era editada por el Dr. Luis Hidalgo y Carpio, se hallan escritos relacionados con la cardioangiología. En el primer tomo, correspondiente al periodo 1864-1865, se encuentran los artículos: De la obliteración de las arterias, escrito por el Dr. M. F. Jiménez (pp. 6-8) y Observación de la ligadura de la arteria axilar a consecuencia de una cornada, del Dr. Aronsohn (pp. 337-338). El tomo ॥ (1866) contiene: Aneurisma inguinal, curación obtenida por ligadura de la iliaca por el Dr. Luis Muñoz, del Dr. E. Liceaga (p. 61) y El yoloxóchitl (Talauma mexicana) árbol cuyas semillas y corteza tienen acción digitálica excelente, de los Dres. G. Mendoza y A. Herrera (p. 223). El tomo III (1867-1868) ofrece 3 artículos de interés cardiovascular: Prueba importante de la percusión y la auscultación en el diagnóstico, de A. Careaga ( $\mathrm{p}$ 235); Las lesiones orgánicas del corazón y de las arterias, de F. Brassetti ( $\mathrm{p}$ 299); y Aneurisma de la aorta, de Lauro M. Jiménez (p. 312). Y el tomo v (1870): Aneurisma traumático difuso de la arteria anónima, de C. Boves (p. 229). En el tomo vIII de 1873 (p. 223) se describen en detalle las características de la digitalina Nativelle. A su vez, el Dr. Ricardo Egea y Galindo, originario de Puerto Rico, relata la primera intervención quirúrgica en el área cardiaca. Se trata de un caso de hidropericardio tratado con éxito por punción evacuadora mediante el aspirador de Potain.

\section{Las primeras investigaciones cardioangiológicas}

Hacia finales del siglo XIX y a principios del siglo $x x$ se llevaron a cabo, en el laboratorio del Instituto Médico Nacional, creado en 1888, algunas investigaciones de farmacología cardiovascular bajo la guía del Dr. Fernando Altamirano (1848-1907), iniciador de estudios farmacológi$\cos$ en México $^{13}$. El índice del tomo ॥, correspondiente al año 1890, de la revista «El Estudio» seminario de ciencias médicas editado por dicho Instituto, menciona una nota acerca del modo de actuar del potasio sobre el corazón (p. 7) y una crónica concerniente a la acción del yoloxóchitl (p. 127). El tomo III, editado en el mismo año, contiene sendas notas en torno a La digital en pediatría (p. 45), Digital y digitalinas (p. 202) y Acción de la cafeína sobre la circulación ( $p$. 203). En el tomo iv de 1891, se halla una lectura, Análisis de las semillas del yoloxóchitl, del Dr. Eduardo Armendáriz (p. 248); así como un artículo original, El yoloxóchitl, por el Dr. Alfonso L. Herrera (p. 133). Y en el tomo ॥ de 1896, hay otro trabajo original de carácter cardiovascular, La tensión sanguínea en México, por el Dr. Daniel Vergara Lope (p. 110). También en la primera parte del tomo । de Datos para la materia médica mexicana de 1894 , se encuentra el 
artículo El yoloxóchitl (Taluma mexicana), por el Dr. José Ramírez (p. 95). Es conveniente recordar que, en el siglo xVI citaron el yoloxóchitl tanto el médico indígena Martín de la Cruz como el historiador franciscano fray Bernardino de Sahagún y el protomédico Francisco Hernández, pero solo este último le atribuyó cierta acción cardiotónica. En sus palabras «Es un árbol grande... tiene flores con figura de corazón... y son muy apreciadas por los indígenas» ${ }^{14}$. Vale la pena preguntarse si este efecto inotrópico positivo se debía exclusivamente al yoloxóchitl o también al cacaoatl (cacao) $)^{15}$.

\section{Acerca del instrumental cardiológico}

En el marco del desarrollo de la cardiología, amerita recordarse la elaboración de instrumentos de uso común en la práctica clínica, y la de alguna doctrina terapéutica, a la luz de criterios epistemológicos. Consideramos en primer término el proceso de la construcción del esfigmomanómetro. Al tomar como modelo el quimógrafo del fisiólogo Karl Ludwig, se crearon en el siglo xix los esfigmomanómetros de K. Vierordt y de E. J. Marey. Este último dio a conocer en 1878 un instrumento capaz de determinar la presión arterial sistémica con el procedimiento de la pletismografía ${ }^{16}$. Angelo Mosso (1866-1910), su discípulo, construyó a su vez 2 aparatos de tamaño reducido. Pero el primero no era bastante sensible y el segundo no era portátil. De todos modos, al emplearlo, se llegó a admitir que, cuando aumentaba la presión en el manómetro, las máximas pulsaciones registradas correspondían a la presión arterial diastólica. Cuando dichas pulsaciones desaparecían, se registraba la presión arterial sistólica. Por su lado, el Dr. Samuel von Basch, quien fuera médico de cámara del archiduque Maximiliano de Habsburgo en México desde el 18 de septiembre de 1866 hasta la muerte de este en Querétaro (junio de 1867), a su regreso a la patria preparó sucesivamente 3 modelos de esfigmomanómetro ${ }^{17}$. Al efectuar numerosas mediciones de la presión arterial, se dio cuenta de la existencia de una entidad patológica definida actualmente como hipertensión esencial a la que él había denominado: «aterosclerosis latente». Gracias a sus estudios y a los de Huchard y de Alibut $^{18}$, se asentó el concepto de que la hipertensión arterial puede deberse a causas distintas a la enfermedad de Bright.

El esfigmomanómetro de columna de mercurio, ideado por Basch en 1880, sirvió de modelo al instrumento construido más tarde por el Dr. Scipione Riva-Rocci. A la luz del pensamiento crítico de Popper, la elaboración de tal instrumento puede considerarse como un problema de tipo epistemológico. En 1896, el Dr. Riva-Rocci, de la Clínica Médica del Hospital Universitario de Turín, presentó a los miembros del Congreso Italiano de Medicina, que se celebraba en aquella ciudad, un nuevo instrumento para medir la presión arterial. El autor de este invento expuso en una publicación ${ }^{19}$ las premisas clinicofisiológicas que le llevaron a esta realización. En sus palabras: «Hemos investigado el nivel y las variaciones de la presión sanguínea arterial con 2 fines esenciales. Ante todo, se trata de conocer el impacto que ejerce la presión sanguínea sobre la pared de los vasos y los tejidos circunvecinos, lo que permite juzgar el grado de resistencia de dichas paredes $y$, en particular, la posibilidad de su rotura. En segundo lugar, debe valorarse la función cardiaca correspondiente con sus repercusiones circulatorias y biológicas. A mi juicio, no se dispone aún ni de procedimientos ni de instrumentos que permitan desarrollar los postulados de la clínica». De hecho, los aparatos construidos hasta aquel entonces carecían de ciertos requisitos fundamentales. Para Riva-Rocci, se necesitaba obtener un instrumento sencillo, portátil, de manejo fácil y de aplicación incruenta. A partir de una teoría que contiene un núcleo de verdad, se hizo posible la elaboración de los instrumentos anteriores. Y el investigador italiano llegó a formular una hipótesis que sería ampliamente confirmada por la experiencia. A saber, dado que el sistema arterial contiene un líquido (la sangre), cuyo movimiento es causado por una bomba (el corazón) y puesto que la presión de dicho líquido depende tanto del trabajo de la bomba como del calibre y de la tensión de las arterias, resulta lógica «la aplicación de principios de hidráulica a la circulación sanguínea concebida como un sistema de tubos comunicantes sometidos al principio de Torricelli» ${ }^{20}$. Con base en el primer modelo de von Basch, modificado posteriormente por Robinson y por Potain, quien sustituyó la transmisión hidráulica por la aérea, Riva-Rocci pudo estructurar felizmente su esfigmomanómetro ${ }^{21}$. Este instrumento, fundamentado en el punto de partida de Vierordt, puede medir de manera manométrica la presión necesaria para impedir la progresión de la onda del pulso. Se compone de 2 partes: una destinada a ejercer la compresión sobre la arteria y otra que permite medir la presión ejercida. Debe tenerse presente que la esfigmomanometría se efectúa ahora sobre la arteria humeral, no sobre la radial, por lo que la medición refleja la carga total en un punto cercano a la aorta. De este modo, tras haber definido claramente el problema por resolver, Riva-Rocci lo examinó desde el punto de vista de la física hidráulica que, en el enfoque lógico, podía proporcionar el modelo más cercano a la realidad.

\section{Contribuciones del Instituto Nacional de Cardiología Ignacio Chávez}

En nuestra época, el 18 de abril de 1944 se inauguró el Instituto Nacional de Cardiología de México, primea institución especializada de atención médica, investigación y enseñanza en el mundo. Poco después algunos de sus miembros presentaron en un foro cardiológico internacional un novedoso procedimiento diagnóstico, que sería de gran utilidad para el estudio cardioangiológico por vía hemodinámica: la cardioangiografía selectiva. Tal procedimiento, ideado por el propio director, Dr. Ignacio Chávez Sánchez, fue aceptado con interés y entusiasmo en el ambiente cardiológico internacional. Los autores anunciaban ${ }^{22}$ que «esta técnica evita la fuga de la sustancia opaca hacia los territorios venosos y reduce al mínimo la mezcla con el torrente sanguíneo, de modo que se obtiene un contraste sumamente intenso de las cavidades cardiacas y, sobre todo, hace posible obtener bellas imágenes de las cavidades cardiacas izquierdas así como de la aorta y de sus ramas, cosa no lograda antes por los métodos usuales». Por otra parte, tenemos un ejemplo significativo del enfoque correcto de un problema terapéutico cardiológico en la concepción de la terapia metabólica de ciertas cardiopatías. Las bases 


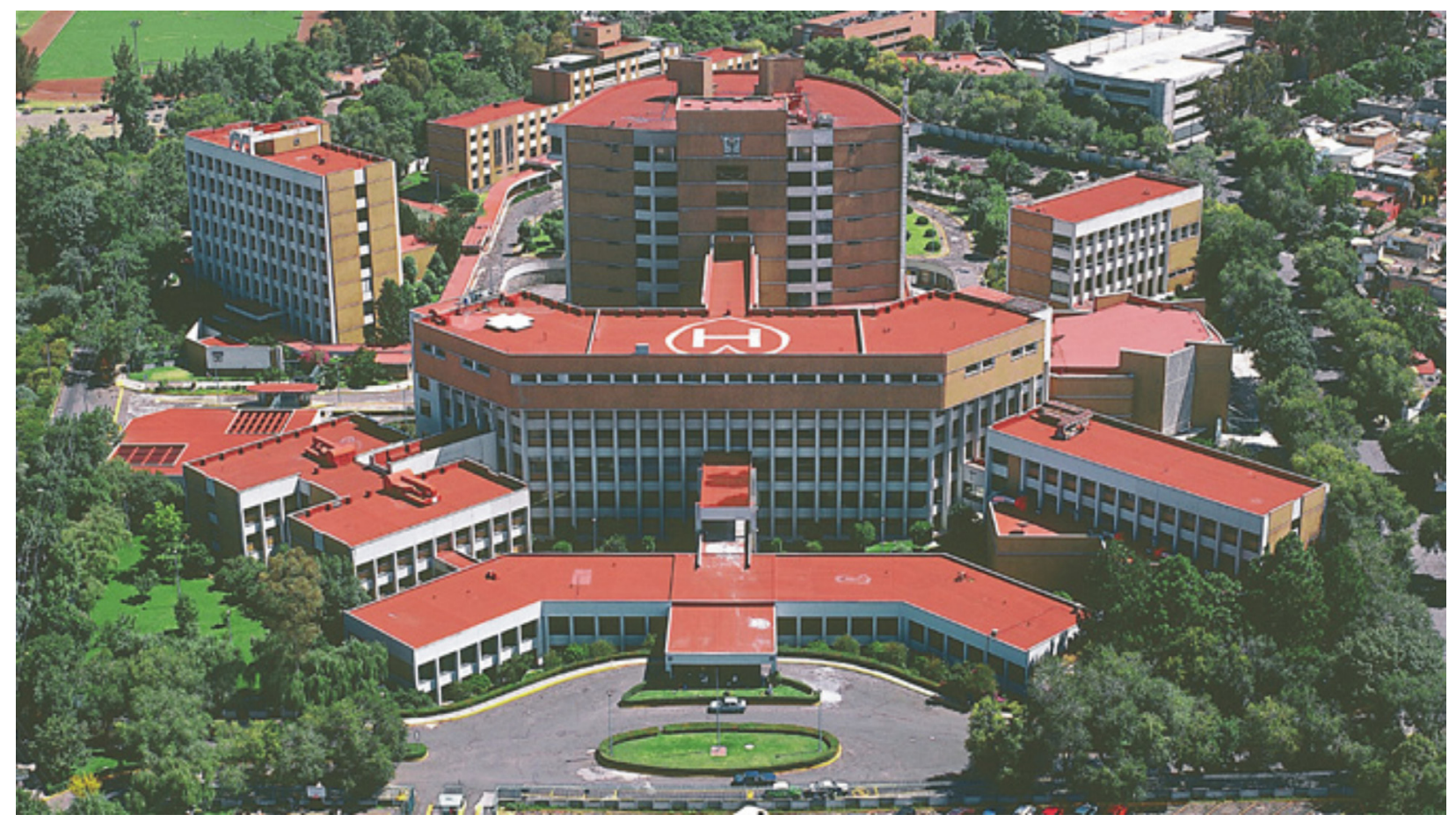

Figura 1 Vista aérea del Instituto Nacional de Cardiología Ignacio Chávez en la Ciudad de México.

de este procedimiento terapéutico se hallan en estudios y aplicaciones relacionadas con el segundo principio de la termodinámica clásica en sistemas fisicoquímicos abiertos y cerrados (Boltzmann, Planck ${ }^{23}$ ) y en los dominios de la biología (Prigogine ${ }^{24}$ y otros). Sobre estas bases se justifica el empleo experimental y clínico de sistemas donadores de energía, p. ej. las soluciones de glucosa, insulina y potasio, en la insuficiencia cardiaca ${ }^{25}$ y en varias cardiopatías ${ }^{26}$. Los resultados obtenidos a nivel celular ${ }^{27}$ y general ${ }^{28}$ parecen demostrar el buen fundamento de la hipótesis inicial y la utilidad del procedimiento mencionado. Más aún, debe tenerse presente que, así como lo demostraron R. Mendez et al. ${ }^{29}$. con la preparación de la doble arritmia en la aurícula derecha de corazón canino, la acción antiarrítmica del potasio se ejerce en aquellas arritmias provocadas o mantenidas por descargas de focos ectópicos de automatismo, mientras que es nula en las que se mantienen por movimiento de circo: flutter y fibrilación. Dichas aportaciones experimentales han constituido un hito de la Escuela Mexicana ${ }^{30}$ en el campo del tratamiento farmacológico de los trastornos del ritmo cardiaco. La insulina, por su lado, actúa sobre la entrada y la formación de ATP en las células miocárdicas. Según ciertos autores $^{31}$, esta hormona ejerce también una acción antiinflamatoria. Conviene mencionar que, desde hace tiempo, se han propuesto las soluciones de glucosa, insulina y potasio como protectoras del miocardio en el síndrome isquemiareperfusión por ser acarreadoras de radicales libres de oxígeno $^{32}$ o por constituir un mecanismo antiapoptósico ${ }^{33}$. $Y$ tal vez podrían utilizarse en los procedimientos de acondicionamiento y postacondicionamiento farmacológicos. En conclusión, las soluciones de glucosa, insulina y potasio siguen utilizándose en cardioangiología pero en dosis más elevadas ${ }^{34}$ que las usadas inicialmente en clínica. Las investigaciones y las enseñanzas de los aspectos mencionados de la cardiología siguen realizándose con interés y entusiasmo en las instalaciones actuales del Instituto Nacional de Cardiología inauguradas en octubre de 1976 (fig. 1).

\section{Financiación}

No se recibió patrocinio de ningún tipo para llevar a cabo este artículo.

\section{Conflicto de intereses}

El autor declara no tener ningún conflicto de intereses.

\section{Bibliografía}

1. Salgado MJ. Cursus Medicus Mexicanus. México: Her. de la Vda. de Miguel de Rivera; 1727. p. 179-217.

2. Fernández del Castillo F. Sor Juana Inés de la Cruz y la medicina de su tiempo. México. Claustro de Sor Juana. Cuaderno n. ${ }^{\circ} 13$, 1981.

3. Díaz de Gamarra Dávalos JB. Elementa recentioris philosophiae. ... México: Impr. de J. A. de Juaregui; 1774.

4. Izquierdo JJ. La fisiología en México. México: UNAM; 1934.

5. Jiménez MF. Lecciones dadas en la Escuela de Medicina de esta capital. Gac Med Mex. 1866;2:257-65, 302-4; 331-6.

6. Delgado García M. Estudio histórico de la hemostasia quirúrgica en México [tesis]. México: Ed. UNAM; 1951.

7. Sénac JB. Traité de la structure du coeur. Paris: Briasson; 1749.

8. Bouillaud J. Traité clinique des maladies du coeur. Paris: J.B. Baillière; 1835.

9. Ruiz LE. Apuntes históricos de la Escuela Nacional de Medicina. México: Ed. UNAM; 1983. p. 58.

10. Carrillo M. Diagnóstico diferencial de las enfermedades del corazón [tesis]. México: Imprenta del Comercio; 1870. 
11. Icaza y Peña JN. Algunas consideraciones acerca de la acción fisiológica de la digital y las indicaciones de su empleo en las afecciones orgánicas del corazón [tesis]. México: Escuela de Medicina; 1872.

12. Martínez R. Estudio sintético sobre la sintomatología de las lesiones valvulares del corazón en sus relaciones con el esfigmógrafo [tesis]. México: Impr. de I. Paz; 1878.

13. Fernández del Castillo F. Historia bibliográfica del Instituto Médico Nacional de México. México: Imprenta Universitaria (UNAM); 1961.

14. Hernández F. Obras completas. México: Ed. UNAM; 1959-1967.

15. Torre JM. La cardiología en el manuscrito de Martín de la Cruz y Juan Badiano. Arch Inst Cardiol Mex. 1979;49:103-13.

16. Marey EJ. La méthode graphique dans les sciences expérimentales et principalement en physiologie et en médecine. París: Ed. Masson; 1878.

17. Delgado García G, Estañol B. El medico imperial antes y después del imperio. Gac Med Mex. 2012;148:487-94.

18. Alibut TC. Senile Plethora on high arterial pressure in elderly persons. Trans Hunter Soc. 1985;77:38ss.

19. Riva-Rocci S. Un nuevo sfigmomanometro. Gaz Med Torino. 1896:50-1, 981-96, 1001-17.

20. Timio M. Scipione Riva-Rocci e la nisurazione della pressione arteriosa, teorie ed applicazioni. Quad St Sci. 1985;2:29ss.

21. De' Caterina V. Un'esemplare applicazione del metodo scientifico: Scipione Riva-Rocci e l'invenzione dello sfigmomanometro a mercurio. Notiziario di Cardiologia dell'ANCE. 1987;: 30-2.

22. Chávez I, Dorbecker N, Celis A. Valor diagnóstico de los angiocardiogramas obtenidos por inyección directa intracardiaca a través de una sonda. Arch Inst Cardiol Mex. 1947;17: 121-54.

23. Planck M. Una autobiografía científica. Ensayos científicos. México: Ed. Conacyt; 1982.
24. Prigogine I. La termodinámica de la vida. Biología molecular. México: Ed. Conacyt; 1981. p. 199-224.

25. Kisthinios N. Le traitement des cardiopathies par l' association sucre-insuline. París: Ed. H. B. Baillière; 1933.

26. Sodi Pallares D, de Micheli A:. Un tentativo di reintegrazione ionica cellulare in alcune malattie cardiovascolari. Atti Acc Med Lomb. 1962;17:509-19.

27. Sybers HD, Maroko PR, Ashraf M, et al. The effect of glucoseinsulin-potassium on cardiac ultrastructures following acute experimental coronary occlusion. Am J Path. 1973;70:401-20.

28. Martínez Rosas $M$, Luna Ortiz P (coord.), Rojas Perez EM, de Micheli A, Pastelín G, Flores P. Terapéutica metabólica en la cardiopatía isquémica. En: Luna Ortiz P, coor. Anestesia cardiovascular. 4. ${ }^{\mathrm{a}}$ ed. Cap. 4. México: Ed. Alfil; 2013. p. 179-197.

29. Mendez R, Aceves J, Kabela E. Differences among quinidine, atropine and potassium in their action on experimental arrythmias. Acta Cardiol. 1965;20:1-12.

30. Mínguez I. Acción antiarrítmica del potasio. En: Bayés A, Cosín J, editores. Diagnóstico y tratamiento de las arritmias cardiacas. Madrid: Ed. Doyma; 1978. p. 744-50.

31. Vlasselaers D, Mesones D, Langanche I, et al. Tight glycemic control protects the myocardium and reduces inflammation in neonatal heart surgery. Ann Thorac Surg. 2010;90:26-9.

32. Hess A, Okabe I, Poland E, et al. J: Glucose, insulin, potassium protection during hypothermic global ischemia and reperfusion: A new proposed mechanism by the surgery of free radicals. J Cardiovasc Pharmacol. 1983;5:35-45.

33. Johanson A, Brian B, Mjos D, et al. Insulin administered at reoxygenation exerts a cardioprotectionary effect in myocites by a possible antiapoptotic mechanism. J Mol Cell Cardiol. 2000;32:757-64.

34. Fan $Y$, Zhang $A M$, Xiao $Y B$, et al. Glucose-insulin-potassium therapy in adult patients undergoing cardiac surgery: A metaanalysis. Eur J Cardiothorac Surg. 2011;40:192-9. 